\title{
Correction to: A modified flux-wave formula for the solution of second order macroscopic traffic flow models
}

\author{
M. Araghi (i) - S. Mahdizadeh - H. Mahdizadeh $\cdot$ S. Moodi
}

Published online: 14 December 2021

(C) Springer Nature B.V. 2021

Correction to: Nonlinear Dyn (2021) 106:3507-3520

https://doi.org/10.1007/s11071-021-06935-w

The original article was published with incomplete author ORCID information. For author, Sara Mahdizadeh, the ORCID information should be noted as:

ORCID: 0000-0001-5954-5409

The original article can be found online at https:// doi.org/10.1007/s11071-021-06935-w.

M. Araghi $(\bowtie) \cdot H$. Mahdizadeh

Department of Civil Engineering, University of Birjand,

Birjand, Iran

e-mail: m.araghi@birjand.ac.ir

H. Mahdizadeh

e-mail: m.araghi@birjand.ac.ir

S. Mahdizadeh

Department of Architecture and Urbanism, Ferdowsi

University of Mashhad, Mashhad, Iran

S. Moodi

Department of Civil Engineering, University of Sistan and

Baluchesatn, Zahedan, Iran
Affiliation: Department of Architecture and Urbanism, Ferdowsi University of Mashhad, Mashhad, Iran

This correction stands to correct the original article.

Publisher's Note Springer Nature remains neutral with regard to jurisdictional claims in published maps and institutional affiliations. 